\title{
Quantum Size Effects And Transport Phenomena In PbSe Quantum Wells And PbSe/EuS Superlattices
}

\author{
E.I. Rogacheva ${ }_{2}^{1}$ O.N. Nashchekina ${ }^{1}$, S.I. Ol'khovskaya ${ }^{1}$, A.Yu. Sipatov ${ }^{1}$, and \\ M.S. Dresselhaus ${ }^{2}$, \\ ${ }^{1}$ National technical university "Kharkov polytechnic institute", 21 Frunze St., Kharkov, 61002, UKRAINE \\ ${ }^{2}$ Massachusetts Institute of Technology, 77 Massachusetts Ave., Cambridge MA 02139, USA
}

\begin{abstract}
It is established that the room-temperature dependences of transport properties on the total thickness of PbSe layers $d$ in $\mathrm{PbSe} / \mathrm{EuS}$ superlattices exhibit an oscillatory behavior. It is shown that the oscillation period $\Delta d$ practically coincides with the period of the thickness oscillations observed earlier in single $\mathrm{PbSe} / \mathrm{EuS}$ quantum well. The non-monotonic character of these dependences is attributed to quantum size effects. The theoretically estimated and experimentally determined $\Delta d$ values are in good agreement.
\end{abstract}

Keywords: PbSe, quantum well, superlattice, transport phenomena, quantum size effects.

PACS: 73.21.-b; 73.50.Jt; 73.50.Lw.

\section{INTRODUCTION}

IV-VI semiconductors are known as promising materials for optoelectronic, thermoelectric, and other applications [1]. These compounds are also convenient objects for studying quantum size effects (QSEs) by measuring transport properties due to a small effective mass and a high charge carrier mobility. In [2], we reported the observation of oscillations in the thickness dependences of the transport properties in $\mathrm{PbSe}$ quantum wells (QWs) and attributed them to the QSEs. Later we observed similar oscillations in other IV-VI QWs (see e.g., $[3,4]$ ).

The goal of this work is to compare the character of the thickness dependences of the transport properties in single $\mathrm{PbSe} \mathrm{QWs}$ and $\mathrm{PbSe} / \mathrm{EuS}$ superlattices (SLs) grown on (001) KCl substrates.

\section{EXPERIMENTAL}

In $[2,5]$ we described in detail the preparation technique and the growth mechanism for $(001) \mathrm{KCl} /$ $\mathrm{PbSe} / \mathrm{EuS} \mathrm{SLs}$. In the present work the thickness of PbSe layers $\left(d_{0}\right)$ in SLs was varied in the range of $d_{0}=$ $0.5-15 \mathrm{~nm}$, whereas the thickness of a EuS layers was $\sim 10 \mathrm{~nm}$. The upper (covering) EuS layer was 30-40 $\mathrm{nm}$ thick. The number of SLs periods was varied from 5 to 25 . All measurements were conducted on freshly prepared samples. The electrical conductivity $\sigma$ and the Hall coefficient $R_{H}$ were measured using $d c$ method, with an error not exceeding $5 \%$. The Hall concentration $n$ and mobility $\mu$ of carriers were calculated as $n=1 /\left(R_{H} \cdot e\right)$ and $\mu=R_{H^{*}} \sigma$, respectively.
The Seebeck coefficient $S$ was measured relative to $\mathrm{Cu}$ with an accuracy of $\pm 3 \%$. All SLs exhibited $n$-type conductivity.

\section{RESULTS AND DISCUSSION}

In Fig. 1, the dependences of $\sigma, n, \mu$, and $S$ on the total thickness $d$ of all PbSe layers $\left(d=N \cdot d_{0}\right)$ for $\mathrm{PbSe} / \mathrm{EuS} \mathrm{SLs}$ are presented. All the dependences exhibit an oscillatory behavior with the mean value of the oscillation period $\Delta d=22 \pm 3 \mathrm{~nm}$. The $\Delta d$ value was determined using the value of $d$ corresponding to the position of the first extremum in the dependences of all kinetic coefficients as well as using the mean distance between the minima and maxima.

In the inserts to Fig. 1, the thickness dependences of the kinetic coefficients for the single (001)/PbSe/EuS QWs obtained in [2] are presented. The oscillation period measured as was indicated above for SLs $(\Delta d=20 \pm 5 \mathrm{~nm})$ is very close to $\Delta d$ for the SLs. The experimental value of $\Delta d$ for single QWs $(\Delta d=35 \pm 3 \mathrm{~nm})$ reported in [2] is an overestimate because $\Delta d$ was determined only from the distance between extrema, which is less accurate in case when the number of studied samples with different thicknesses is limited.

One can assume that the observed oscillatory behavior of the $d$-dependences of the transport properties both in $\mathrm{PbSe} / \mathrm{EuS} \mathrm{SLs}$ and in single $\mathrm{PbSe} / \mathrm{EuS} \mathrm{QWs}$ can be interpreted in terms of quantum-mechanical concepts about a gradual filling of the lateral subbands with increasing $d$. The difference between QWs and SLs consists in the fact 

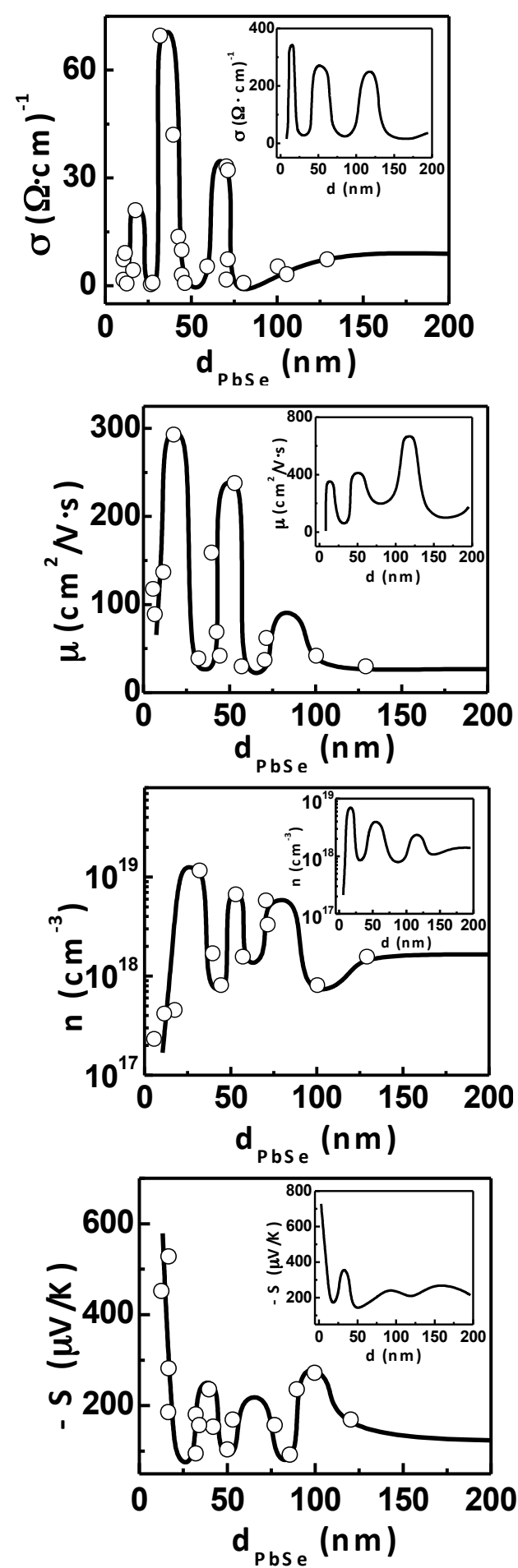

FIGURE 1. Dependences of the electrical conductivity $\sigma$, Seebeck coefficient $S$, electron mobility $\mu$, and concentration $n$ on total PbSe layers thickness $d$ in $\mathrm{PbSe} / \mathrm{EuS}$ superlattices. In the inserts: the dependences of $\sigma, \mu, n$, and $\mathrm{S}$ on thickness for single $\mathrm{PbSe} / \mathrm{EuS}$ quantum wells reported in [2].

that in SLs one should take into account the total thickness of all PbSe layers $\left(d=N \cdot d_{0}\right)$, which can be changed either by changing the PbSe layer thickness $\left(d_{0}\right)$ or by changing the number of periods $(N)$. It is taken into account that the conductivity in the studied SLs occurs in the interface planes and that the barrier width is sufficiently small $(10 \mathrm{~nm})$ for the realization of the tunneling processes. Every time $d$ increases by $\lambda_{F} / 2$, where $\lambda_{F}$ is the de Broglie wavelength at the Fermi level $\varepsilon_{F}$, an additional subband drops below $\varepsilon_{F}$, and contributes to electrical conduction. One can estimate the oscillation period $\Delta d$ (see [2-5]) using the following equation:

$$
\Delta d=\frac{\lambda_{\mathrm{F}}}{2}=\frac{h}{\sqrt{8 \mathbf{m}_{\mathrm{z}}^{*} \varepsilon_{\mathrm{F}}}}
$$

where $m_{z}{ }^{*}$ is the effective mass for motion perpendicular to the QWs. The critical thickness $d_{1}$, at which the first subband passes through the Fermi level $\left(E_{l}=\varepsilon_{F}\right)$, can be estimated as $d_{l}=h / \sqrt{ } 8 m_{z}{ }^{*} \varepsilon_{F}=\Delta d$. Knowing effective masses of the electrons in $n$-PbSe $\left(m_{\mathrm{t}}{ }^{*}=0.04 m_{0}, m_{1}=0.07 m_{0}\right.$ [1]) and estimating $\varepsilon_{F}$ from the average electron concentration $\left(n \approx 2 \cdot 10^{18}\right.$ $\mathrm{cm}^{-3}$ ), one can calculate $\Delta d$ using Eq. 1, thus obtaining $\Delta d=20 \pm 5 \mathrm{~nm}$, which is in good agreement with the experimentally determined values of $\Delta d$ for QWs $(\Delta d$ $=20 \pm 5 \mathrm{~nm}[2])$ and for SLs $(\Delta d=22 \pm 3 \mathrm{~nm})$.

Thus, the experimentally determined periods of the quantum thickness oscillations of the transport properties in $\mathrm{PbSe} / \mathrm{EuS}$ single QWs and $\mathrm{PbSe} / \mathrm{EuS} \mathrm{SLs}$ practically coincide and correspond to the results of theoretical calculations.

\section{ACKNOWLEDGMENTS}

The Ukrainian team was supported by the Ukrainian State Fund for Basic Research (grant \# UU 42/006 2011). The MIT author acknowledges support from NSF/DMR-11-07339.

\section{REFERENCES}

1. Yu.I. Ravich, B.A. Efimova, I.A. Smirnov, Semiconducting Lead Chalcogenides, Plenum Press (New York, 1970), pp. 156-227.

2. E.I. Rogacheva, T.V. Tavrina, O.N. Nashchekina, S.N. Grigorov, K.A. Nasedkin, M.S. Dresselhaus, and S.B. Cronin, Appl. Phys. Letters 80, 2690-2693 (2002).

3. E.I. Rogacheva, O.N. Nashchekina, A.V. Meriuts, and Lyubchenko, M.S. Dresselhaus, G. Dresselhaus, Appl. Phys. Letters 86, 063103 (2005).

4. E.I. Rogacheva, O.S. Vodorez, O.N. Nashchekina, A.Yu. Sipatov, A.G. Fedorov, S.I. Olkhovskaya, M.S. Dresselhaus, J. Electr. Materials 39, 2085-2091 (2010).

5. E.I. Rogacheva, O.N. Nashchekina, A.Yu.Sipatov, A.G.Fedorov, S.N.Grigorov, T.V.Tavrina, and M.S. Dresselhaus, Phys. Stat. Solidi C 6, 1149-1154 (2009). 
AIP Conference Proceedings is copyrighted by AIP Publishing LLC (AIP). Reuse of AIP content is subject to the terms at: http://scitation.aip.org/termsconditions. For more information, see http://publishing.aip.org/authors/rights-and-permissions. 